\title{
Revista Brasileira de Enfermagem REBEn \\ Atitudes de enfermeiros de hospital geral frente ao uso do álcool e alcoolismo
}

\author{
Clinical nurses' attitudes toward the use of alcohol and alcoholism
}

Actitudes de enfermeros de hospital general frente al uso de alcohol y al alcoholismo

Divane Vargas

Doutorando do Departamento de Enfermagem Psiquiátrica e Ciências Humanas da EERP-

USP.

devargas@eerp.usp.br

Renata Curi Labate

Professora Doutora do Departamento de Enfermagem Psiquiátrica e Ciências Humanas da EERP-USP.
Submissão: 04/02/2005

Aprovação: 04/10/2005

\section{RESUMO}

Estudo psicométrico, utilizando a escala Seaman Mannello Nurse's attitudes toward alcohol and alcoholism, aplicou o instrumento a uma população de 196 enfermeiros de um macro-hospital, visando a mensurar as atitudes de enfermeiros de hospital geral no que se refere ao álcool e ao beber. Os dados apontaram que os enfermeiros consideram que as bebidas alcoólicas são prejudiciais $(54,4 \%)$, beber com moderação não é inofensivo( $57,1 \%)$ e é errado $(47,4 \%)$. Revelam dificuldade na aceitação do beber como um direito da pessoa, considerando as bebidas alcoólicas capazes de tornar pessoas saudáveis "débeis e loucas" (29,8\%). 0 enfermeiro é um profissional indispensável para o tratamento e recuperação do alcoolista, suas atitudes podem influenciar no relacionamento com o paciente e conseqüentemente favorecer o tratamento.

Descritores: Alcoolismo; Consumo de bebidas alcoólicas; Atitude do pessoal de saúde; Enfermeiro.

\section{ABSTRACT}

This psychometric study used the Seaman Mannello's scale of "nurse's attitudes toward alcohol and alcoholism". Authors applied an instrument to a population of 196 nurses from a general hospital with the aim to measure nurses' attitudes toward alcohol and drinking. Data showed that nurses consider alcoholic drinks clearly prejudicial (54.4\%), that moderately drinking is not innofensive (57.1\%) and drinking is wrong (47.4\%), demonstrating that it is difficult to accept drinking as a person right. $29.8 \%$ of the sample considered alcoholic drinks able to change healthy people into "disturbed and demented" ones. Authors concluded that the nurse is an essential professional in the treatment and rehabilitation of alcoholics. Nurses attitudes can influence the relationship with the patient and, consequently, favor the treatment.

Descriptors: Alcoholismo; Alcohol drinking; Attitude of health personnel; Nurse.

\section{RESUMEN}

En este estudio psicométrico fue utilizada la escala Seaman Mannello Nurse's attitudes toward alcohol and alcoholism y aplicado un instrumento a una población de 196 enfermeros de un macro-hospital, con el objetivo de mensurar las actitudes de enfermeros de hospital general frente al alcohol y beber. Los datos mostraron que los enfermeros consideran que las bebidas alcohólicas son perjudiciales $(54,4 \%)$, beber con moderación no es inofensivo $(57,1 \%)$ y es errado $(47,4 \%)$, revelando dificultad en la aceptación del beber como un derecho de la persona. 29,8\% consideraron las bebidas alcohólicas capaces de tornar las personas saludables en "locas y débiles". El enfermero es un profesional fundamental para el tratamiento y recuperación del alcoholista. Sus actitudes pueden influenciar el relacionamiento con el paciente y, así, favorecer el tratamiento.

Descriptores: Alcoholismo; Consumo de bebidas alcohólicas; Actitud del personal de salud; Enfermero.

Vargas D, Labate RC. Atitudes de enfermeiros de hospital geral frente ao uso do álcool e alcoolismo. Rev Bras Enferm 2006 jan-fev; 59(1):47-51.

\section{INTRODUÇÃO}

$\mathrm{O}$ alcoolismo constitui um dos mais graves problemas médico-sociais dos grandes centros na atualidade. Esse fato tem preocupado governos, profissionais da saúde e a sociedade de um modo geral. O primeiro levantamento domiciliar sobre o uso de drogas psicotrópicas no Brasil, que envolveu as 107 maiores cidades do país ${ }^{(1)}$ apontou que 0 uso de álcool pela população atinge uma porcentagem de $68,7 \%$. Esse resultado está próximo aos $70,8 \%$ observados no Chile e aos $81,0 \%$ constatados nos EUA. O mesmo levantamento apontou que, nas cidades estudadas, a estimativa de dependentes de bebidas alcoólicas era de $11,2 \%$.

Sabe-se que 0 alcoolismo acarreta sérios problemas, dentre eles: problemas sociais, econômicos e de saúde, sendo que as repercussões do uso abusivo na saúde do indivíduo acabam tornando comum o atendimento de pacientes dependentes do álcool nos hospitais gerais, o que faz essa prática freqüente entre os enfermeiros de setores clínicos e cirúrgicos. Este fato é possível de se 
observar ao se analisarem vários estudos ${ }^{(2-5)}$ que trazem informações sobre a internação de alcoolistas nos hospitais especializados e gerais do Brasil.

A experiência prática e a literatura( ${ }^{(5-7)}$ revelam, no entanto, que apesar da crescente demanda de dependentes do álcool nos hospitais, nem sempre o enfermeiro encontra-se preparado para atuar frente a essa problemática. Talvez não seja tarefa fácil para os enfermeiros generalistas compreenderem alguns aspectos que envolvem o alcoolismo, principalmente, porque pouca atenção tem sido dada à questão do abuso de álcool na formação desses profissionais ${ }^{(8,9)}$.

Concomitantemente com a dificuldade em compreender o alcoolismo em suas várias dimensões, associa-se o fato de o alcoolismo constituirse em uma doença estigmatizada pela sociedade, podendo essa questão influenciar as atitudes dos enfermeiros que cuidam de pacientes alcoolistas, através das opiniões das pessoas, experiências vividas, crenças, valores e conhecimentos.

O enfermeiro é um dos profissionais mais adequados ao tratamento das doenças mentais e particularmente 0 alcoolismo, sendo que suas atitudes têm um grande impacto na relação com o paciente e conseqüentemente nos resultados do tratamento ${ }^{(10)}$.

Por ser o enfermeiro o profissional que tem o maior contato com o paciente alcoolista durante a internação, o relacionamento entre eles deve favorecer a construção de um ambiente que possa influenciar a decisão do paciente em facilitar o tratamento ${ }^{(11)}$. A percepção que 0 enfermeiro tem do paciente é o principal determinante da qualidade, bem como da quantidade do cuidado de enfermagem que será prestado $^{(12)}$. Quando o enfermeiro possui uma atitude negativa, o paciente pode, em contrapartida, desenvolver uma postura hostil ou contra-terapêutica. Sendo assim, uma análise das atitudes desses profissionais é necessária, uma vez que essas atitudes poderão interferir no relacionamento e tratamento do alcoolista.

Outra razão importante para estudar atitudes de enfermeiros frente ao álcool e ao alcoolismo deve-se ao fato de o hospital geral constituirse, na maioria das vezes, porta de entrada para o tratamento dos problemas relacionados ao álcool(6).

Diante da influência das atitudes do enfermeiro no relacionamento e tratamento do alcoolista, bem como das dificuldades que permeiam essa relação, desenvolveu-se um estudo para mensurar as atitudes do enfermeiro de hospital geral no que se refere ao álcool e aos problemas com o beber

\subsection{Conceituando atitudes}

Concepções clássicas de atitude surgiram nos livros de Psicologia na década de $1970^{(13,14)}$. As ações sociais do indivíduo refletem sua atitudes, as quais são sistemas duradouros de avaliações positivas e negativas, sentimentos emocionais e tendências de ação, favoráveis e desfavoráveis, com relação a objetos sociais ${ }^{(14)}$.

Uma atitude é a predisposição adquirida e duradoura a agir sempre do mesmo modo diante de uma determinada classe de objetos, ou um persistente estado mental e/ou neural de prontidão para reagir diante de uma determinada classe de objetos, não como eles são, mas sim como são concebidos ${ }^{(13)}$.

\section{OBJETIVO}

Verificar as atitudes de enfermeiros de hospital geral frente ao álcool e ao beber.

\section{METODOLOGIA}

Estudo psicométrico que utilizou, como instrumento de mensuração, o Seaman Mannello Nurse's attitudes toward alcohol and alcoholism $S c a l e^{(15)}$. Esse instrumento foi criado e validado pelas autoras, com uma amostra original de 439 enfermeiras, em Buffalo, New York, EUA. É composto por 30 itens, divididos em cinco subescalas: trata-se de um instrumento específico para mensurar atitudes de enfermeiros frente ao álcool e ao alcoolismo .

$O$ presente estudo trata da subescala $V$ que mede as atitudes pessoais dos enfermeiros frente ao álcool e ao beber. Os sujeitos da pesquisa posicionaram-se frente aos itens através de uma escala do tipo likert com opções de resposta de 1 a 5 pontos, sendo que 1 representou completo desacordo com o item proposto e 5 completo acordo. $O$ quadro 1 apresenta os itens da subescala $V$, os quais foram respondidos pelos sujeitos.

\begin{tabular}{|l|l|}
\hline \multicolumn{2}{|c|}{ Itens da subescala V } \\
\hline 01 & $\begin{array}{l}\text { A bebida alcoólica em quantidade moderada pode na verdade não } \\
\text { prejudicar a saúde. }\end{array}$ \\
\hline 02 & $\begin{array}{l}\text { Não há nada de errado com beber quantidades moderadas de } \\
\text { álcool. }\end{array}$ \\
\hline 03 & Bebidas alcoólicas são inofensivas quando usadas com moderação. \\
\hline 04 & As pessoas devem beber bebidas alcoólicas se elas desejarem. \\
\hline 05 & $\begin{array}{l}\text { Quando usadas moderadamente, bebidas alcoólicas são tão } \\
\text { inofensivas, quanto bebidas não alcoólicas. }\end{array}$ \\
\hline 06 & $\begin{array}{l}\text { O consumo de bebidas alcoólicas não pode fazer pessoas normais, } \\
\text { débeis e loucos. }\end{array}$ \\
\hline
\end{tabular}

\section{Quadro 1. Itens que compõe a subescala V, Atitudes pessoais frente ao álcool e o beber.}

A população do estudo constituiu-se de 196 enfermeiros de um macro-hospital geral de um município do interior paulista, dos quais $88 \%$ eram do sexo feminino, cinco eram diretoras de seção $(2,9 \%)$; quinze enfermeiros-chefes $(8,8 \%)$; dois enfermeiros encarregados $(1,2$ $\%$ ) e 149 enfermeiros $(87,1 \%)$. A idade variou entre 22 e 51 anos, e $51 \%$ dos sujeitos revelaram experiência profissional com alcoolistas.

0 instrumento foi entregue em envelopes lacrados e foi solicitado que os questionários não fossem identificados, a fim de garantir a privacidade dos mesmos. Dos 196 questionários entregues, 171 (82,2\%) foram devolvidos.

O questionário levou aproximadamente 30 minutos para ser respondido e foram inclusas no mesmo as instruções para o preenchimento, bem como o termo de consentimento esclarecido.

A análise foi dividida em duas etapas. Inicialmente, os dados coletados foram lançados no EPINFO, programa da Organização Mundial da Saúde (OMS), de domínio público, utilizado para análise de estudos dessa natureza. Isso possibilitou realizar uma análise descritiva das informações sócio-demográficas da população estudada.

Posteriormente, analisaram-se as questões das cinco subescalas que compõem o The Seaman Mannello Nurses'attitudes Toward Alcohol and alcoholism, atribuindo 1 e 2 pontos para categorias de respostas desfavoráveis ao item; três para categorias intermediárias e quatro e cinco pontos para categorias favoráveis. Da soma dos escores obtidos, calculou-se uma média, sendo esse resultado interpretado, de acordo com as autoras do instrumento.

Para fins de apresentação neste estudo, agruparam-se os itens 1discordo totalmente e 2- discordo em 1-discordo , 2- não concordo nem discordo (neutralidade) e 4 concordo e 5- concordo totalmente em 3- concordo.

\section{ANÁLISE E DISCUSSÃO DOS RESULTADOS}

A média do escore obtido nessa subescala que mede as atitudes dos enfermeiros frente ao álcool e ao beber foi de 16,08, escore considerado baixo. Os enfermeiros deste estudo vêem o álcool como perigoso, sendo nocivo em qualquer quantidade e moralmente errado(15).

Para apresentação dos resultados, dividiu-se a análise em duas categorias. Na primeira, são apresentados e discutidos os dados referentes às atitudes pessoais dos enfermeiros sobre a bebida alcoólica e, na segunda, os dados referentes às atitudes frente ao 
consumo das mesmas.

\subsection{Atitudes pessoais frente à bebida}

A análise dos resultados dos itens que avaliam as atitudes dos enfermeiros frente à bebida alcoólica mostra que, entre as enfermeiras participantes do estudo, predominam atitudes pessoais negativas com respeito ao álcool. Verificou-se que mais da metade da população estudada $(54,4 \%)$ considera que as bebidas alcoólicas, mesmo que usadas moderadamente, prejudicam a saúde, $57,1 \%$ acreditam que o beber com moderação não é inofensivo. Enquanto $66,1 \%$ consideram que a ingestão moderada de bebidas alcoólicas não é tão inofensiva quanto a ingestão de bebidas não alcoólicas.

As figuras que seguem mostram a distribuição das respostas frente aos itens que mensuram as atitudes pessoais no tocante às bebidas alcoólicas.

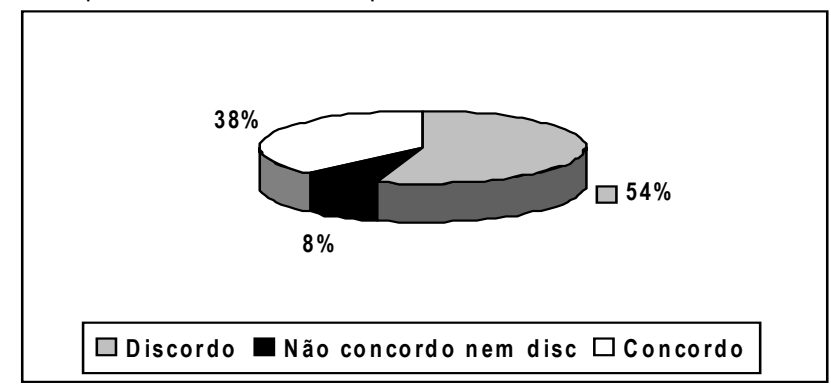

Gráfico 1. A bebida alcoólica em quantidades moderadas pode, na verdade, não prejudicar a saúde.

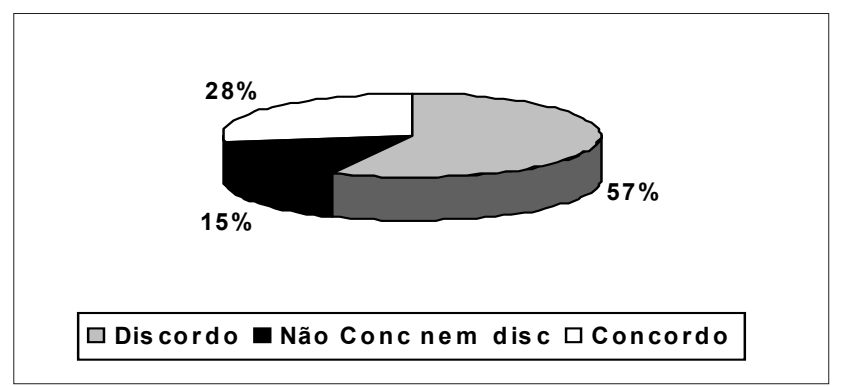

Gráfico 2. Bebidas alcoólicas são inofensivas quando usadas com moderação.

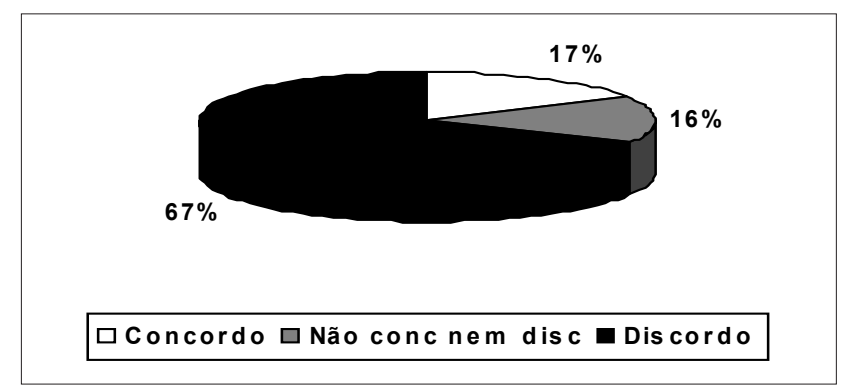

Figura 3. Quando usadas moderadamente, bebidas alcoólicas são tão inofensivas quanto bebidas não alcoólicas.

Com relação às conseqüências do álcool, os dados apontaram que os enfermeiros percebem nas bebidas alcoólicas um perigo para a saúde de quem as consome, não importando a quantidade consumida. Talvez esse resultado esteja relacionado às concepções e vivências proporcionadas por experiências desses enfermeiros ao prestarem assistência a pacientes com complicações decorrentes do beber. Associado a essa questão, é possivel, ainda, que as informações recebidas durante a graduação em disciplinas de formação geral, que enfatizam o álcool como fator contribuinte e, muitas vezes, desencadeante de uma série de patologias orgânicas, poderiam justificar tal achado.
Por outro lado, as dificuldades encontradas, no tratamento do alcoolismo, podem contribuir para essas atitudes, uma vez que os enfermeiros entram em contato com esse paciente em estágios já muito avançados da doença, quando as complicações clínicas se manifestam e quando, na maioria das vezes, tornam-se irreversíveis. Daí que a questão da formação, da vivência e da constatação dos resultados provocados pelo uso do álcool pode levar esses enfermeiros a considerar a bebida nociva e perigosa para a saúde do indivíduo, justificando suas atitudes negativas frente à bebida alcoólica.

Cabe ressaltar, no entanto, que as atitudes dos enfermeiros contra o beber parecem não ser tão seguras. Quando precisam avaliar a nocividade do uso moderado do álcool, percebe-se que não existe uma atitude totalmente definida, pois $54,4 \%$ dos enfermeiros acreditam que o beber mesmo moderadamente prejudica a saúde, no entanto, quase $40 \%$ dos enfermeiros acreditam que o beber moderado não é prejudicial e somente $7,6 \%$ dos entrevistados colocaram-se em posições intermediárias, sem opinião formada.

\subsection{Atitudes frente ao beber}

$\mathrm{Na}$ questão que avaliou as atitudes pessoais dos enfermeiros frente ao beber, os resultados encontrados revelam a não-aceitação do beber por parte dos entrevistados. O beber é encarado como errado (Gráfico 4), sendo que as pessoas deveriam evitar tal prática (Gráfico 5), pois acreditam que 0 álcool é capaz de tornar o indivíduo débil e louco (Gráfico 6).

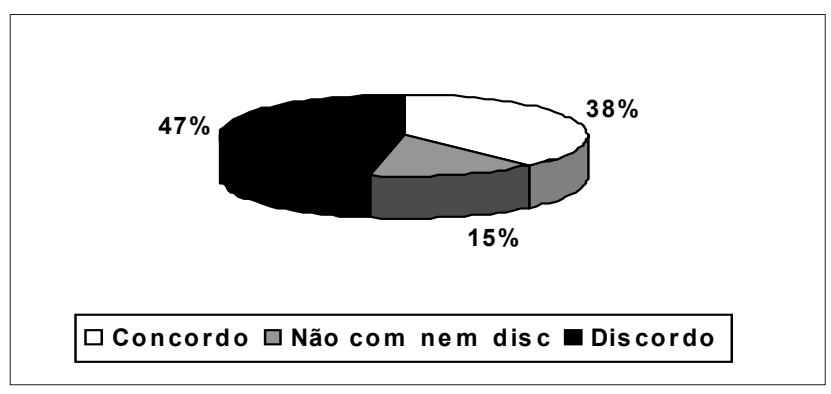

Gráfico 4. Não há nada de errado em beber quantidades moderadas de álcool.

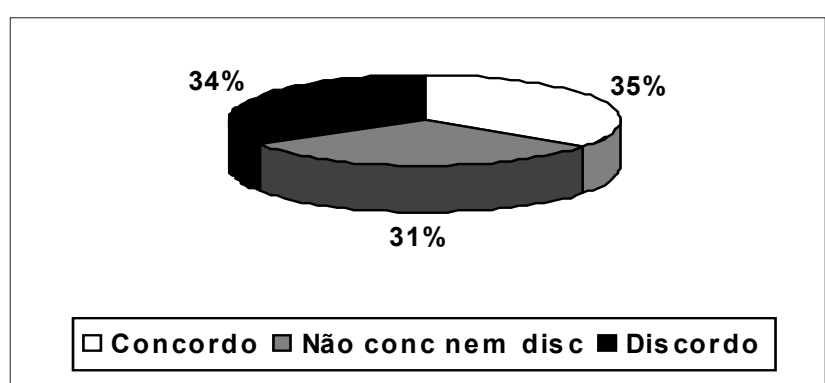

Gráfico 5. As pessoas devem beber bebidas alcoólicas se elas desejarem.

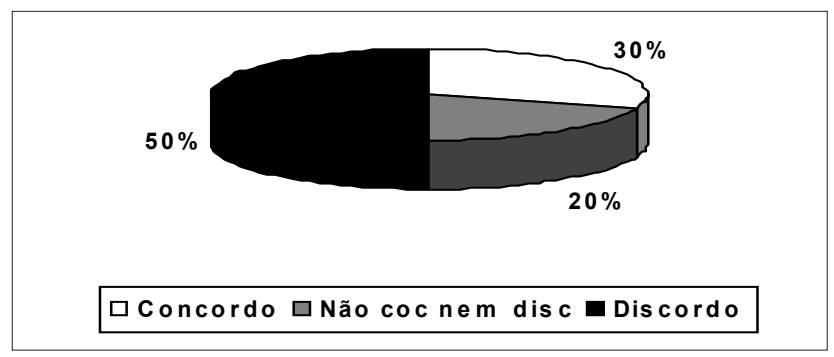

Gráfico 6.0 consumo de bebidas alcoólicas não pode fazer pessoas normais débeis e loucas. 
Ao encararem o beber como algo moralmente errado, é possível que atribuam à doença a vontade própria do paciente que, mesmo conhecendo as conseqüências do uso do álcool, age de maneira errada ao consumi-lo.

Embora $47 \%$ dos enfermeiros consultados encarem o beber moderado como algo errado, chama atenção o fato de um percentual bem próximo a esse (38\%) acreditar não existir nenhum problema em consumir bebidas alcoólicas. Tal achado talvez se justifique pelo fato de que muitos enfermeiros refletem a visão dominante da sociedade, que aceita 0 beber moderado e rejeita 0 indivíduo que bebe de maneira descontrolada ${ }^{(16,17)}$.

Dados semelhantes foram encontrados em estudo realizado no norte da Inglaterra ${ }^{(17)}$. A razão apontada pelos autores para hesitação nas atitudes das enfermeiras frente ao beber foi atribuída à aceitação difundida socialmente do beber moderado, bem como à comparação com o próprio consumo de álcool das enfermeiras.

Ao considerarem o beber como moralmente errado, é possível que atribuam ao alcoolista o caráter da voluntariedade da doença. Sabe-se que, dentre as patologias psiquiátricas, o alcoolismo é uma das mais rejeitadas, sendo 0 alcoolista estigmatizado por seu problema ${ }^{(18)}$.

A retirada do caráter de voluntariedade da ingestão de bebidas alcoólicas abole, ao menos oficialmente, o julgamento moralista em relação aos dependentes do álcool, segundo o qual a responsabilidade de ingestão exagerada de bebidas alcoólicas é atribuída à degradação moral(19).

Em um estudo realizado com 140 estudantes de enfermagem ${ }^{(20)}$, encontrou-se que os mesmos preferiam cuidar de pacientes que se mostrassem dependentes e comunicativos e mostraram-se menos aptos a trabalhar com pacientes que na sua concepção fossem responsáveis pela sua doença. Sendo assim, ao considerar o uso do álcool sobre o cunho moral, os estudantes podem também atribuir a responsabilidade da doença ao próprio indivíduo quando ele perde o controle.

Em um outro estudo(21), verificou-se que estudantes de enfermagem tinham menos atitudes de aceitação frente ao alcoolista do que frente a pacientes fisicamente incapacitados. É possível que a não-aceitação do alcoolista esteja relacionada à questão do consumo exagerado de bebidas alcoólicas, nesse sentido a dependência pode ser vista como um sintoma da doença provocada pela prática errada do beber.

Os enfermeiros também revelam dificuldade na aceitação do beber como um direito da pessoa, manifestando-se uns, de maneira radical, favoravelmente $(35 \%)$ ou contra (34\%) e outros sem opinião formada (31\%).

Uma revisão de estudo de atitudes de profissionais para com o alcoolismo sugere falta de aceitação do mesmo, sendo que vários grupos de profissionais reconhecem 0 alcoolismo. Sendo, porém, mais fácil aceitar isso intelectualmente do que emocionalmente ${ }^{(22)}$.

$\mathrm{Na}$ opinião da metade dos enfermeiros do estudo ( $50 \%)$, as bebidas alcoólicas são capazes de tornar pessoas saudáveis em "débeis e loucas". As alterações do psiquismo atribuídas aos efeitos do álcool sobre o funcionamento do sistema nervoso parecem justificar tal visão.

Pois a baixa tolerância à frustração, em referência às reações inadequadas e pouco ajustadas, frente a estímulos desagradáveis, justifica o modo de o alcoólico encarar a realidade dos outros, negando a sua realidade e alterando a percepção da realidade do outro. Isso seria a conseqüência da mudança fácil e repetida da consciência sobre 0 efeito da bebida(23).

Além disso o humor lábil do paciente alcoólico, manifestado muitas vezes, por irritação, descontrole, ansiedade e depressão sem causas concretas, pode ser associado a sintomas de debilidade ou loucura.

Tal fato é preocupante, pois sugere dificuldade em entender esses sintomas e condutas como decorrência da dependência, atribuindo-os à doença mental. É possível que o alcoolismo seja classificado enquanto doença mental, devido ao fato de que, como já mencionado anteriormente, os enfermeiros têm recebido pouca informação e treinamento específico sobre 0 alcoolismo ${ }^{(8,9)}, 0$ que reflete a pouca atenção dada à temática na formação dos profissionais de saúde, tornando 0 conhecimento incipiente ${ }^{(9)}$.

\section{CONSIDERAÇÕES FINAIS}

De acordo com os resultados obtidos neste estudo, os enfermeiros consideram que as bebidas alcoólicas prejudicam a saúde $(54,4 \%)$, que o beber com moderação não é inofensivo( $57,1 \%)$ e é errado $(47,4 \%)$. Consideram ainda que a ingestão moderada de bebidas alcoólicas não é tão inofensiva quanto a ingestão de bebidas não alcoólicas $(66,1 \%)$. Além disso revelam dificuldade na aceitação do beber como um direito da pessoa, manifestando-se uns, de maneira radical, favoravelmente $(36,9 \%)$ ou contra $(34,5 \%)$ e outros sem opinião formada $(31,5 \%)$. Também consideram que as bebidas alcoólicas são capazes de tornar pessoas saudáveis em "débeis e loucas" $(29,8 \%)$.

A média encontrada nessa subescala $(16,08)$ é considerada desfavorável e revela atitudes negativas. Um escore baixo como o obtido neste estudo indica que, na concepção do enfermeiro, o álcool é perigoso, sendo assim seu consumo é nocivo independentemente da quantidade ingerida e é moralmente errado(15).

Isso leva a pressupor que, sob o ponto de vista dos enfermeiros deste estudo, o álcool é perigoso e nocivo, mesmo que usado socialmente, e que seu uso pode ser visto por esses profissionais como moralmente errado. Sendo assim, podem atribuir ao indivíduo as causas e conseqüências de sua doença, uma vez que podem conceber que 0 paciente conhece os riscos oferecidos pelo uso do álcool.

Faz-se necessária a retirada do caráter de voluntariedade da ingestão de bebidas alcoólicas, abolindo o julgamento moralista em relação aos dependentes do álcool, segundo o qual a responsabilidade de ingestão exagerada de bebidas alcoólicas é atribuída à degradação moral do indivíduo (19)

Ver o alcoolismo como algo moralmente errado permite rotular 0 alcoolista como fraco e dependente, culpando-o pela sua fraqueza de caráter. 0 pressuposto de que 0 alcoolista pode controlar seu hábito de beber tem influenciado a população de um modo geral(25). É possível que o enfermeiro sofra influências do meio social. A relação da sociedade com o álcool é contraditória, pois ao mesmo tempo em que existe aceitação de seu consumo, observa-se a reprovação severa ao alcoolismo propriamente dito, que é sempre associada a uma decadência moral, econômica, volitiva do indivíduo e que acaba por levá-lo à rejeição social(22).

Talvez isso justifique o fato de $40 \%$ dos enfermeiros do estudo apresentarem atitudes favoráveis frente ao beber moderado, mesmo que $70 \%$ deles considerem as bebidas alcoólicas prejudiciais em qualquer quantidade.

O dependente de álcool não deve ser visto como alguém que cometeu uma falha moral, mas como vítima de uma doença cujo sinal patogênico é a perda do controle individual e comunitário(19).

Sendo assim, é importante que o enfermeiro examine suas atitudes acerca do alcoolismo e ofereça cuidados sem julgamento à pessoa do alcoolista, haja vista que é responsável em várias situações pelos cuidados prestados a esses pacientes. Para isso faz-se necessário 0 reconhecimento das fontes de suas atitudes; explorar como suas atitudes afetam os cuidados prestados ao paciente complementando com autoconsciência e mudança de certas atitudes comprovadas como negativas. Entende-se que 0 alcoolismo é uma problemática constante nos locais de trabalho dos enfermeiros. $O$ enfermeiro é um profissional capacitado a atuar com esse paciente, tendo em vista que tem a vantagem de poder assistir o paciente de forma mais ampla e em várias situações acompanhando, aconselhando e ajudando o paciente a atingir 
a estabilidade, além disso é o profissional que se encontra em uma das posições mais privilegiadas para entender os sentimentos dos pacientes, segundo diferenças sociais, culturais e individuais ${ }^{(18)}$.

Diante disso, o enfermeiro é um profissional indispensável para o tratamento e a busca da recuperação do alcoolista e suas atitudes podem influenciar definitivamente no relacionamento com o paciente alcoolista e conseqüentemente favorecer o tratamento.

Lançar mão de uma prática mais humana, onde exista mais sensibilidade de escuta destituída de preconceitos pode ser um primeiro passo para garantir uma assistência de qualidade a essa clientela. Tal prática poderá ser viabilizada com o estabelecimento de três fatores fundamentais e inter-relacionados: mudança de atitudes, busca de conhecimentos e aperfeiçoamento de habilidades. Essa conscientização dos profissionais se faz necessária, uma vez que os dados mostram que cada vez mais o paciente alcoolista faz parte do cotidiano do enfermeiro generalista.

\section{REFERÊNCIAS}

1. Carlini EA, Galduróz JCF, Noto AR, Nappo AS. I Levantamento domiciliar sobre o uso de drogas psicotrópicas no Brasil: estudo envolvendo as 107 maiores cidades do país: 2001 São Paulo (SP): CEBRID - Centro Brasileiro de Informações Sobre Drogas Psicotrópicas : UNIFESP - Universidade Federal de São Paulo; 2002.

2. Masur J, Cunha J, Zuiker A.P. Prevalência de pacientes com indicadores de alcoolismo internados em uma enfermaria de clínica geral: relevância da forma de deteç̧ão. Acta Psiq Psicol da América Latina 1980; $125-30$

3. Diniz SA. Sentido da vida base para a compreensão de alcoolista [dissertação]. Ribeirão Preto (SP): Escola de Enfermagem de Ribeirão Preto, Universidade de São Paulo; 1992.

4. Raeder JR, Carline-Cotrin B. Internações hospitalares no Brasil por dependência de drogas, álcool e psicoses alcoólicas, em 1988. Rev ABP-APAL 1990; 12(1/4): 33-9.

5. Bertolote JM. Alcoolismo hoje. $3^{\text {a }}$ ed. Porto Alegre (RS): Artes Médicas; 1997.

6. Bertolote JM, Lemos NLT. Alcoolismo: sua detecção no hospital geral. Acta Med 1983: 290-5

7. Figlie NB, Pillon SC, Laranjeira RR, Dunn J. "O AUDIT identifica a necessidade de interconsulta específica para dependentes do álcool no Hospital geral?" J Bras Psiq 1997 nov-dez; 48 (11): 589-93.

8. Ramos LH, Pillon SC, Cavalcante MBG, Luis MAV, Padredi FM, Laranjeira RR. $O$ ensino sobre dependência química em cursos de graduação em enfermagem no Brasil, 1998. Acta Paul Enferm 2001 set-dez; 14(3): 35-43.

9. Luis MAV, Pillon SC. O conhecimento dos alunos de Enfermagem sobre álcool e drogas. Rev Eletrônica Enferm 2003; 5(1): 21-7.

10. Sowa PA, Cutter HS. Attitudes of hospital staff toward alcoholics. and drug addicts. J Stud Alcohol 1974 jan-mar; 35: 210-4.

11. Gross BV, Lisman S. Attitudes of paraprofessssionals toward alcoholism; setting effects. J Stud Alcohol 1979; 40( 5).

12. Travelbee J. Interpersonal aspects of nursing. Philadelphia (USA): Davis; 1971.

13. Krech DC, Ballachey RS, Egerton L. O Individuo na sociedade: um manual de psicologia social. Sao Paulo (SP): Pioneira, 1969.

14. Rodrigues A. psicologia social. $12^{\mathrm{a}}$ ed. Petrópolis (RJ): Vozes; 1988.

15. Seaman J, Mannello T. Nurses' attitudes toward for alcohol and alcoholism- the Seaman Mannello scale. Rockville (USA): National Institute on Abuse and Alcoholism; 1978.

16. Allen K. Attitudes of registered nurses toward alcoholic patients in a general hospital population. Intern J Addictions 1993; 28(9): 923-30.

17. Lock CA, Kaner E,Lamont S, Bond S. A qualitative study of nurse's and pratices regarding brief alcohol intervention in primary health care. J Advanced Nurs 2002; 39(4): 333-42.

18. Gil-Merlos AS. Aceitação e rejeição do alcoolismo: um estudo com alunos de enfermagem [dissertação]. Ribeirão Preto (SP): Escola de Enfermagem de Ribeirão Preto, Universidade de São Paulo;1985.

19. Ramos SP, BertoloteJM, organizadores. Alcoolismo hoje. $3^{\mathrm{a}}$ ed. Porto Alegre (RS): Artes Médicas; 1997.

20. Bae E. Lowery B. Patient and situtional factors that affect nursing studentes' like or deslike of caring for patients. Nurs Res 1987; 36: 298-302.

21. Shimid J. Shimid D. Nursing students' attitudes toward alcoholics. Nurs Res 1973; 22: 246-8.

22. Vargas HS. Repercussões do álcool e do alcoolismo. São Paulo (SP) Byk-Procienx; 1983.

23. Dractu L. Araujo VA. Alcoolismo: do conceito ao tratamento. J Bras Psiq 1985 jul-ago; 34(4): 263-8.

24. Masur J, Jorge M.R. Dados relacionados às bebidas alcoólicas e alcoolismo no Brasil: uma revisão. Rev ABP-APAL 1986; 8(4):157-65. 